\title{
Itinerário terapêutico: olhar atento sobre a Atenção Primária a Saúde no interior da
}

\author{
Amazônia \\ Therapeutic itinerary: a closer look at Primary Health Care in the interior of the Amazon \\ Itinerario terapéutico: una mirada más cercana a la Atención Primaria de Salud en el interior de la
}

Amazonía

Recebido: 04/10/2021 | Revisado: 13/10/2021 | Aceito: 21/10/2021 | Publicado: 23/10/2021

\author{
Camila dos Santos Freitas \\ ORCID: https://orcid.org/0000-0003-1540-8319 \\ Universidade Estadual do Pará, Brasil \\ E-mail: camilafreitas0208@gmail.com \\ Vanessa Mello da Silva \\ ORCID: https://orcid.org/0000-0002-6272-9732 \\ Universidade Estadual do Pará, Brasil \\ E-mail: vtavares1973@hotmail.com \\ Georgia Silvestri Traesel \\ ORCID: https://orcid.org/0000-0002-8042-5791 \\ Universidade Estadual do Pará, Brasil \\ E-mail georgia_traesel@ hotmail.com \\ Franciane de Paula Fernandes \\ ORCID: https://orcid.org/0000-0002-4617-1919 \\ Universidade Estadual do Pará, Brasil \\ E-mail: franciane.fernandes@uepa.br \\ Sheyla Mara Silva de Oliveira \\ ORCID: https://orcid.org/0000-0001-6666-2363 \\ Universidade Estadual do Pará, Brasil \\ E-mail: sheylaoliveira@uepa.br \\ Marcelo Silva de Paula \\ ORCID: https://orcid.org/0000-0003-0320-0051 \\ Universidade Estadual do Pará, Brasil \\ E-mail: marcellodipaula86@gmail.com \\ Lívia de Aguiar Valentim \\ ORCID: https://orcid.org/0000-0003-4255-8988 \\ Universidade Estadual do Pará, Brasil \\ E-mail: livia.valentim@uepa.br \\ Gabriel Cunha da Silva \\ ORCID: https://orcid.org/0000-0002-1051-7674 \\ Universidade Estadual do Pará, Brasil \\ E-mail: gabriel.csilva@aluno.uepa.br
}

\begin{abstract}
Resumo
O estudo tem por objetivo investigar o itinerário terapêutico na Atenção Primária à Saúde na modalidade da Estratégia Saúde da Família. Trata-se de um estudo descritivo, de abordagem qualitativa, constituindo-se uma pesquisa de campo realizada no município de Santarém - PA. Participaram da pesquisa, os usuários e profissionais que atuam em duas Estratégia Saúde da Família (ESF), sendo uma localizada na zona urbana e outra na zona do rural. Para coleta de dados, utilizou-se um roteiro de perguntas, que levou em consideração características sociodemográficas dos participantes. Os dados foram agrupados segundo a análise de conteúdo categorial de Bardin. A pesquisa respeitou a resolução 466/2012 do Conselho Nacional de Saúde e foi submetido Junto ao Comitê de Ética em Pesquisa em Seres Humanos da Universidade do Estado do Pará (UEPA) sob CAAE: 20314619.7.0000.5174 e parecer de aprovação número: 3.619.147. Os resultados das entrevistas, foram organizados segundo as percepções dos profissionais de saúde e usuários em relação aos eixos temáticos estudados: 1: itinerário na promoção a saúde e na busca do tratamento de doenças; 2: potencialidades e fragilidades encontradas no acesso ao serviço. Constatou-se que a UBS é o primeiro local de procura pelos usuários, quando estão doentes. Quanto ao eixo temático 2, o agendamento de consultas é tido como uma das potencialidades da ESF, entretanto, o número reduzido de profissionais, caracterizou-se como uma das fragilidades assistenciais. Assim, por meio desta pesquisa percebeu-se que os itinerários terapêuticos são característicos de cada indivíduo, baseados em suas experiências e crenças.
\end{abstract}

Palavras-chave: Itinerário terapêutico; Atenção primária à saúde; Práticas de saúde. 


\begin{abstract}
The study aims to investigate the therapeutic itinerary in Primary Health Care in the modality of the Family Health Strategy. This is a descriptive study, of qualitative approach, constituting a field research conducted in the municipality of Santarém - PA. Participated in the research, users and professionals who work in two Family Health Strategies (FHS), one located in the urban area and the other in the rural area. For data collection, we used a questionnaire that took into account the participants' sociodemographic characteristics. The data were grouped according to Bardin's categorical content analysis. The research complied with resolution 466/2012 of the National Health Council and was submitted to the Ethics Committee on Human Research of the Universidade do Estado do Pará (UEPA) under CAAE: 20314619.7.0000.5174 and approval opinion number 3.619.147. The results of the interviews were organized according to the perceptions of health professionals and users in relation to the thematic axes studied: 1: itinerary in health promotion and in the search for treatment of diseases; 2: potentialities and weaknesses found in access to the service. It was found that the UBS is the first place that users seek when they are sick. Regarding thematic axis 2, the scheduling of appointments is seen as one of the potentialities of the FHS; however, the reduced number of professionals was characterized as one of the weaknesses in assistance. Thus, through this research it was realized that the therapeutic itineraries are characteristic of each individual, based on their experiences and beliefs.
\end{abstract}

Keywords: Therapeutic itinerary; Primary health care; Health practices.

\title{
Resumen
}

El estudio pretende investigar el itinerario terapéutico en Atención Primaria en la modalidad de la Estrategia de Salud Familiar. Se trata de un estudio descriptivo de enfoque cualitativo, constituyendo una investigación de campo realizada en el municipio de Santarém - PA. Participaron en la investigación usuarios y profesionales que se encontraban en dos Estrategias de Salud de la Familia (ESF), una localizada en la zona urbana y otra en la zona rural. Para la recogida de datos se utilizó un guión de preguntas que tenía en cuenta las características sociodemográficas de los participantes. Los datos se agruparon según el análisis de contenido categórico de Bardin. La investigación cumplió con la resolución 466/2012 del Consejo Nacional de Salud y fue sometida al Comité de Ética en Investigación en Seres Humanos de la Universidad del Estado de Pará (UEPA) bajo el CAAE: 20314619.7.0000.5174 y el dictamen de aprobación número 3.619.147. Los resultados de las entrevistas se organizaron según las percepciones de los profesionales de la salud y de los usuarios en relación con los ejes temáticos estudiados: 1: itinerario en la promoción de la salud y en la búsqueda de tratamiento de las enfermedades; 2: potencialidades y debilidades encontradas en el acceso al servicio. Se comprobó que el SBU es el primer lugar de búsqueda de los usuarios cuando están enfermos. En cuanto al eje temático 2, la programación de citas se considera una de las potencialidades del FSE, sin embargo, el reducido número de profesionales se caracterizó como una de las debilidades en la asistencia. Así, a través de esta investigación se constató que los itinerarios terapéuticos son característicos de cada individuo, basados en sus experiencias y creencias.

Palabras clave: Itinerario terapéutico; Atención primaria de salud; Prácticas sanitarias.

\section{Introdução}

O termo itinerário terapêutico é utilizado para definir o percurso percorrido por pessoas em busca de cuidados terapêuticos não necessariamente coincidentes com esquemas ou fluxos pré-determinados. É definido por todo o percurso em saúde, seja ele em prevenção ou de recuperação desta e envolvem cuidados caseiros, práticas religiosas até práticas médicas. Escolher o caminho a percorrer durante busca de assistência à saúde expressa condições sob influência de diversos contextos: subjetivos individuais e coletivos, acerca do processo de saúde e doença (Cabral et al. 2011).

Dessa forma, a pessoa que adoece perpassa um itinerário de cuidado da saúde em busca de orientações, explicações e tratamento para sua doença. Deve-se compreender então que a doença, segundo Kleinmam (1980), é um processo cujas manifestações dependem de fatores sociais, culturais e psicológicos.

A estrutura interna do sistema de saúde é descrita, segundo Kleinmam (1980) como um sistema cultural formado por três subsistemas inter-relacionados: o profissional, dotado de profissionais de cura legalmente reconhecidos; o subsistema popular, de conhecimento não profissional e leigo, por onde as doenças são primariamente identificadas; e o subsistema folclórico, especialista em cura, porém não profissionalmente habilitados. Através dessas três ordens, os indivíduos constroem sua percepção de doença, tratamento e cura (Visentin, 2010).

Devido aos fatos expostos acima, a informação sobre em que momento e circunstancias as pessoas procuram ajuda para resolver suas necessidades de saúde são importantes para a avaliação de planejamento e organização dos sistemas de 
assistência (Cabral et al. 2011).

Os estudos e investigações sobre itinerários terapêuticos são relativamente recentes. A investigação sobre o tema possibilita aos profissionais da área da saúde conhecerem a percepção da pessoa doente sobre sua patologia, como ela vive e reage diante dos sinais, sintomas e da incapacidade provocada pela doença (Alves \& Souza, 1999).

Tendo, portanto, o principal foco de interesse o juízo do paciente sobre a doença e tratamento e o fato de que estudo sobre itinerários terapêuticos podem ser importantes ferramentas para a qualificação da assistência e contribuem para a construção de práticas assistenciais mais compreensivas e contextualmente integradas (Alves \& Souza, 1999).

Diante do exposto, este estudo tem por objetivo investigar o itinerário terapêutico na Atenção Primária à Saúde na modalidade da Estratégia Saúde da Família.

\section{Metodologia}

Tipo de Estudo: Estudo descritivo, de campo com abordagem qualitativa.

O estudo possui abordagem qualitativa, descritiva, constituindo-se de uma pesquisa de campo. Para Minayo (2010), o método qualitativo "é o que se aplica ao estudo da história, das relações, das representações, das crenças, das percepções e das opiniões, produtos das interpretações que os humanos fazem a respeito de como vivem, constroem seus artefatos e a si mesmos, sentem e pensam". Desse modo, essa abordagem permite analisar as percepções dos indivíduos acerca de determinado objeto. Segundo Fernandes et al. (2018), uma pesquisa descritiva consiste em um trabalho que oferece margens para a explicação das relações de causa e efeito dos fenômenos, além de permitir ao pesquisador a obtenção de uma melhor compreensão do comportamento de diversos fatores e elementos que influenciam determinado fenômeno.

A pesquisa de campo já é caracterizada pelas investigações em que, além da pesquisa bibliográfica e/ou documental, se realiza a coleta de dados junto ao público alvo do estudo (Temer \& Tuzo, 2017).

Caracterização do Local do Estudo: O Município de Santarém está situado ao norte do Brasil, na Mesorregião do Baixo Amazonas, microrregião de Santarém e constitui-se do centro polarizador da Região Oeste do Pará - área que abrange 722.358 $\mathrm{km}^{2}$ e abriga vinte e sete municípios. Segundo o Instituto Brasileiro de Geografia e Estatística (IBGE), o município possui 306.480 habitantes (IBGE, 2021).

Está inserido no $9^{\circ}$ Centro Regional de Saúde (CRS), o qual abrange mais de 14 municípios que compõem a região do baixo amazonas, no oeste do Estado do Pará. A rede pública de atenção de Santarém disponibiliza 93 Centros de Saúde distribuídos em Unidades Básicas de Saúde (UBS), seis Unidades Básicas 24 h (UBS-24) e uma Unidade de Pronto Atendimento 24h (UPA-24), o Pronto Socorro (PSM), e ainda os serviços do Hospital Municipal (HMS) e Hospital Regional do Baixo Amazonas (HRBA) (SEMSA, 2020).

Esta pesquisa foi realizada em duas Estratégias Saúde da Família (ESF) sendo uma da zona urbana e outra na zona rural. Os participantes do estudo são profissionais que atuam na ESF, e usuários do serviço de saúde da ESF do município de Santarém. Foram incluídos no estudo profissionais de saúde que compõem a equipe da ESF da zona urbana ou rural; tempo de atuação profissional (há no mínimo um ano); ambos os sexos, que sejam cadastrados no código nacional de estabelecimentos de saúde (CNES) dessas unidades; e os que aceitaram participar do estudo.

Para os usuários do serviço de ESF foram incluídos aqueles que residem no município, maiores de 18 anos e de ambos os sexos. Foram excluídos do estudo os profissionais que não aceitaram participar da pesquisa, ou que por outro motivo não estavam presentes na coleta de dados. Foram também excluídos os usuários residentes de outros municípios da região e que não aceitaram participar da pesquisa. 
Coleta de dados: Neste estudo, para coleta de dados foi utilizado um roteiro de entrevista construído pelas pesquisadoras, bem como serão consideradas variáveis de importância demográfica e epidemiológica: idade, sexo, atividade laboral, renda, escolaridade. O questionário compreende uma relação de perguntas que devem ser respondidas por escrito pelos participantes (Carlomagno, 2018).

Análise de Dados: Os dados advindos das entrevistas, após a transcrição, foram examinados a partir da análise de conteúdo categorial proposta por Bardin (2011). Segundo as três (03) fases de análise: pré-análise, exploração do material, tratamento dos resultados obtidos e interpretação. Apresentam-se de grande relevância à pesquisa sobre sentimentos, pensamentos, saberes, crenças, opiniões de conteúdo construído em relação a determinado fenômeno estudado. Essa análise é destinada à obtenção de categorias de textos que se assemelham e diferem entre si.

A primeira etapa consistiu-se em sistematizar o texto e fazer uma leitura superficial para o reconhecimento do material das entrevistas transcritas. Na segunda, houve um aprofundamento da leitura realizada, a fim de identificar o que era mais significativo para este estudo, assim reagrupando os textos de entrevistas por aproximações de questões que estavam dispersas no texto. Na terceira etapa, os materiais reagrupados foram discutidos para se tornarem válidos à pesquisa (Bardin, 2011).

Assim sendo, esta modalidade de análise se aplica a discursos ou falas de uma maneira bastante diversificada, oscilando entre o rigor da objetividade e a fecundidade da subjetividade, promovendo uma desocultação da mensagem a ser analisada (Bardin, 2011). A técnica de análise de conteúdo desmembra o texto em unidades e o aproxima em categorias reagrupadas analogicamente.

Aspectos Éticos: Este estudo teve como base a Resolução 466/2012 do Conselho Nacional de Saúde, que dispões acerca da pesquisa com seres humanos, respeitando os princípios da autonomia, beneficência, não maledicência, justiça, e equidade dentro outros, e visando assegurar os direitos e deveres que dizem respeito aos participantes da pesquisa, à comunidade científica e ao Estado. Foi submetido junto ao Comitê de Ética em Pesquisa em Seres Humanos da Universidade do Estado do Pará (UEPA) sob CAAE: 20314619.7.0000.5174 e parecer aprovado sob o Número do Parecer: 3.619.147.

Os participantes do estudo foram informados sobre os objetivos da pesquisa e posteriormente assinaram o Termo de Consentimento Livre e Esclarecido (TCLE), assegurando-lhe direito à privacidade e ao sigilo de seus dados e identificações pessoais, como também lhe garantindo a liberdade de se retirar do estudo no momento de sua escolha. Todos os dados foram lançados e armazenados em planilhas eletrônicas por meio dos softwares Microsoft Office Excel 2013 e 2010 (Windows), e nestes permanecerão guardados confidencialmente por cinco anos para que, de acordo com os preceitos éticos, consiga-se salvaguardar as informações pessoais dos participantes deste estudo.

\section{Resultados e Discussão}

Os resultados das entrevistas realizadas nesta pesquisa, foram organizados de acordo com as visões dos profissionais de saúde e dos usuários, sendo agrupados em Eixo Temático 1: itinerário na promoção a saúde e na busca do tratamento de doenças e Eixo Temático 2: potencialidades e fragilidades encontradas no acesso ao serviço.

\section{EIXO TEMÁTICO 1: Itinerário na promoção a saúde e na busca do tratamento de doenças}

Analisando todas as falas dos profissionais, é possível perceber que esse itinerário é complexo, podendo dar destaque a quatro caminhos principais: a procura ao sistema de atenção básica/ESF; a busca direta às unidades de pronto atendimento 
(UPA) e Hospitais; a automedicação e a tentativa de tratamento por conselhos da comunidade, amigos e familiares. Segundo o discurso dos profissionais, quando questionados sobre onde os usuários geralmente vão quando ficam doentes ou precisam de orientação em relação a saúde, foi possível identificar que a maioria procura primeiramente a UBS para assistência.

Este achado também está em consonância com as falas dos usuários entrevistados, quando indagados sobre onde procuram ajuda quando estão doentes, a maioria relatou ir primeiramente na unidade de saúde do bairro, por questões de proximidade geográfica ou até mesmo em prontos atendimentos de maiores complexidades, como hospitais e UPA. Apesar disso, alguns usuários procuram outros locais e opções para o tratamento de sua doença, como a orientação de familiares e amigos, que estão relacionadas a dieta, ervas medicinais, apoio espiritual e emocional.

EIXO TEMÁTICO 2: Potencialidades e Fragilidades encontradas no acesso ao serviço sob a visão dos Profissionais e usuários

O Quadro 1 destaca os achados relacionados ao eixo temático 2.

Quadro 1. Potencialidades e Fragilidades encontradas no acesso ao serviço sob a visão dos Profissionais e Usuários, em Santarém-PA, 2020.

\begin{tabular}{|c|c|}
\hline POTENCIALIDADES & FRAGILIDADES \\
\hline \multicolumn{2}{|c|}{ Profissionais } \\
\hline Número de consultas & Dificuldade para marcar exames \\
\hline Agendamento & Referência e contra referência \\
\hline Visita Domiciliar & Número de profissionais reduzido \\
\hline Longitudinalidade & Falta de insumos \\
\hline Proximidade da UBS com a Residência & Falta de medicamentos \\
\hline \multicolumn{2}{|c|}{ Usuários } \\
\hline Bom atendimento & Falta de insumos \\
\hline Agendamentos & Falta de medicamentos \\
\hline \multirow[t]{2}{*}{ Vínculo com os profissionais } & Número de profissionais reduzido \\
\hline & Falha na cobertura da ESF \\
\hline
\end{tabular}

Fonte: Dados da pesquisa.

\section{Itinerário na promoção a saúde e na busca do tratamento de doenças}

Por meio do relato dos profissionais de saúde, percebeu-se que a busca pelo itinerário terapêutico foi realizada por quatro caminhos principais: atenção básica, a busca direta às UPA's e hospitais, a automedicação e a busca por concelhos de pessoas próximas. Sendo a atenção primária, o caminho percorrido com maior frequência.

A Atenção Básica - AB compõe o primeiro nível de atenção, sendo configurada como porta de entrada ao sistema de saúde, estabelecendo acesso preferencial à população e é capaz de resolver mais de $80 \%$ dos problemas de saúde (Paes, Nascimento \& Oliveira, 2018).

Estudos apontam que há maior utilização da UBS quando há proximidade geográfica. De forma semelhante, Arakawa et al. (2012) afirma em seu estudo sobre a satisfação dos usuários quanto a ESF, que a proximidade é um dos aspectos fundamentais da atenção primária. A acessibilidade organizacional também tem grande impacto na procura pelos clientes por esse nível de assistência, como o horário de funcionamento das Unidades, tempo de espera e agendamentos de 
consultas, bem como obstáculos na realização de procedimentos e o mecanismo de referência-contra referência (Santos et al. 2011; Bizinelli et al. 2019).

Uma parcela menor de profissionais indagados, porém, relatou, como demonstrado nas falas abaixo, que parte dos usuários por vezes procuram, como segundo caminho para busca de tratamento, diretamente serviços mais complexos de atendimento, como UPA e Hospitais considerados "porta de entrada" ao sistema por pacientes classificados não urgentes, em detrimento do primeiro nível de atenção: as Unidades Básicas de Saúde/Estratégia Saúde da Família.

“A gente orienta pra eles virem pra cá, procurar a UBS. E geralmente tem uns que se recusam a vim na UBS e vão direto pra outros locais, tipo o Municipal, eles... as vezes nem é pra lá, né, o problema, as vezes pode ser resolvido por aqui. A gente orienta em primeiro lugar procure a UBS, né, faça um atendimento, se não conseguir eles mesmos lhe encaminham pra UPA ou pra outro local"

"Deveria ser no posto, deveria..., mas não funciona, não funciona na prática. Alguns fazem, outros não. Já procuram direto no hospital ou UPA. Ai na UPA, manda voltar pro posto (risos)."

Nesse contexto, a procura pela UPA em situações urgentes se dá pelo fato de essas unidades apresentarem recursos como consultas, exames, medicamentos e procedimentos de enfermagem, além da proximidade da residência dos usuários ou até mesmo por não haver vagas na UBS. Tais dados sugerem a existência de dificultadas no acesso e tratamento nas unidades de saúde. Vale destacar também que, os usuários relatam como motivo pela procura, maior resolutividade, qualidade e agilidade nos atendimentos, quando comparados a UBS (Santos et al. 2014; Paes, Nascimento \& Oliveira, 2018).

Em relação ao terceiro caminho identificado, a automedicação foi frequentemente mencionada:

"É... e tipo assim a gente orienta muito, bate muito na tecla com eles. A gente tenta orientar de várias formas, pra ver se vai..., mas diminuiu bastante até, com essa proibição, né? A gente vê que diminuiu muito."

Esses dados são consonantes com estudo de Ascari et al. (2014) sobre essa prática por usuários da ESF evidenciando que uma porcentagem significativa dos usuários da ESF pratica a automedicação. Entre os principais fatores que influenciam esse comportamento está a facilidade e o hábito de ir à farmácia e esta fornecer o medicamento sem exigência de receita.

Em relação ao quarto caminho identificado na fala dos profissionais entrevistados, tem-se a procura por parte dos usuários por caminhos não baseados na visão biomédica, encaixados, segundo esquema proposto por Kleinman (1980) sobre cuidados em saúde, na visão popular.

"Inicialmente buscam conselhos de amigos da família, alguns vão diretamente a ESF e outros praticam automedicação.”

O contexto descrito pelos profissionais de saúde quanto ao percurso do itinerário terapêutico também está em conformidade com a visão de grande parte dos usuários entrevistados, que responderam ir preferencialmente à unidade de saúde, quando estão adoentados, ou até mesmo em serviços de maior complexidade como a UPA e o hospital, por ser mais próximo de sua residência ou possuir maior resolutividade.

"Ir ao médico né? Ir ao médico independente de onde seja, onde for mais fácil ou mais rápido, tiver. Geralmente quando é assim em bairro tem que ir logo ao posto médico, ou então no caso se for urgente né, ao pronto socorro."

Apesar disso, alguns usuários procuram outros locais e opções para o tratamento de sua doença, como a orientação de familiares e amigos, que estão relacionadas a dieta, ervas medicinais, apoio espiritual e emocional. 
Os itinerários se organizam em três subsistemas, o familiar relacionado as orientações recebidas, como repouso, dieta, remédios caseiros, suporte emocional e espiritual, de pessoas que possuem vínculos afetivos com o indivíduo; Popular, envolve especialistas de cura não formais que fazem uso de ervas medicinais, exercícios especiais e rituais de curam; e o subsistema profissional, o qual concentra os profissionais da saúde legalmente reconhecidos (Maliskai \& Padilhaii, 2007).

Nessa pesquisa, se evidenciam tais influências.

"Orientações para se tratar? Sempre tem. Uns tios mais velhos... Ou então a minha mãe sempre fala uma história de um mel que a minha tia faz de folha grossa, de malva, mel... É, como é?... Copaiba (risos). Aí mistura, as vezes eu faço."

“Já recebi orientações, mas nem sempre eu sigo. As vezes sim. Tem sempre a automedicação né? Por exemplo tá com dor de garganta, toma nimesulida”

Considerando-se o questionamento anterior, este estudo procurou entender se os entrevistados exercem as práticas que lhe são ensinadas, a maioria referiu a automedicação como primeira medida tomada:

"Ai meu deus, a primeira atitude? Tem que saber, depende do que tá sentindo né? Se for uma febre, toma um antitérmico, observa se vai ter febre de novo; se for, sei lá, uma dor na costa, vai passar um gel pra... Antiinflamatório pra fazer a massagem na costa."

Tal evidência também é confirmado por Merino e Marcon (2007), o qual refere em seu estudo que mais de $40 \%$ da população analisada pratica a automedicação, recorrendo uma espécie de sistema informal de saúde. Ainda de acordo com esse autor, a segunda atitude prevalente nos usuários é a busca por serviços de saúde, evidenciado também o perfil desta pesquisa. Para Guerin (2012) o não cumprimento do itinerário terapêutico formal pode afastar a pessoa do uso adequado do medicamento e consequentemente, comprometer a sua saúde.

\section{Potencialidades e fragilidades encontradas no acesso ao serviço}

No cotidiano dos profissionais e usuários as falas dos profissionais carregam grande contribuições e remetem às limitações bem como as potencialidades encontradas no acesso aos serviços de saúde das duas ESF estudadas.

Quando se trata de Potencialidades os discursos se atém a citar questões relacionadas à organização dos atendimentos conferida pelos agendamentos, deixando um número diário de consultas para as demandas espontâneas; além da realização de visitas domiciliares multiprofissionais e à oportunidade conferida à UBS em realizar um atendimento longitudinal em saúde. Outro benefício citado foi a proximidade geográfica da UBS da residência dos usuários.

Nesse contexto, podem ser citadas como potencialidades na atenção básica, a afinidade com a assistência/cuidado prestado, resolutividade da assistência oferecida aos usuários, prática do acolhimento e veículo com os usuários (Barros et al. 2018).

A insatisfação dos profissionais que atuam na ESF é influenciada pelas condições do trabalho, pela fragilidade na gestão e por problemas nas relações que permeiam o processo de trabalho em saúde, como por exemplo a falta de insumos para execução de procedimentos, dificultando a dinâmica e a resolutividade do serviço de saúde. Além disso, a falha do sistema de referência e contra referência é tida também como um motivo de insatisfação dos profissionais. (Milanez et al. 2018; Albuquerque et el. 2013).

Analisando a percepção dos usuários quanto a essa temática, a maioria referiu como potencialidade um bom atendimento recebido. Pode-se notar o estabelecimento de um vínculo entre eles e os profissionais das ESF’s estudadas. 
"pra mim é muito bom, nota dez né, elas sabem até meu nome, que é o principal né..., chega aqui nós conversa, me dão aquele carinho né, entendeu, não tenho o que me queixar daqui não.”

"Ah, aqui, assim, eu não tenho nada que reclamar, né. Porque toda vez que eu procuro, sempre sou atendida, sempre eles dão atenção. Não tenho o que reclamar não."

O vínculo entre profissionais e usuários é de grande relevância na atenção à saúde dos indivíduos e no processo de saúde-doença. Partindo disso, o modelo de atenção preconizado pelo PSF é entendido a partir do espaço físico e social que a população se encontra, assim, é possível o melhor entendimento de tal processo e levantamento dos problemas de saúde naquela região, buscando resolvê-los, por meio de uma rede de saúde intersetorial com enfoque no atendimento de qualidade, resolutivo e humanizado (Guedes, Santos \& Lorenzo, 2011; Formiga, 2016).

Outra potencialidade apontada foi a eficácia no agendamento de consultas, como se observa nos trechos de entrevistas abaixo:

“..., eu to achado bom agora que a consulta a gente vem e agenda ne? e vem. Porque teve uma época que tinha que vir pra cá de madrugada tirar ficha, tinha gente que dormia pra pegar senha pra tirar ficha. aí eu acho melhor assim, a gente vem e agenda, e vem pra consulta."

Apesar de a agilidade no agendamento de consultas ser constatado nesta pesquisa, outros estudos apontam que há uma demora para se conseguir tal consulta, levando este usuário a buscar outras alternativas para resolução do problema. Nesse sentido, esse acontecimento pode refletir numa baixa resolutividade das demandas apresentadas pelos usuários (Mendes, 2010; Corrêa et al. 2011; Marin et al. 2014).

Alguns usuários entrevistados tiveram como relato negativo a carência de insumos e de medicamentos na ESF.

"o material já faltou algumas vezes aqui, mas eu comprei e deixei aqui né, porque não ia levar pra casa, mas o pessoal não tem culpa, é que falta mesmo né..."

A falta frequente de medicamentos é um dos fatores que dificultam o bom funcionamento dos serviços executados pelos profissionais de saúde da ESF, comprometendo o controle de doenças tais como, hipertensão e diabetes.

"As vezes falta medicação da pressão e eu tenho que comprar pra não ficar sem”

Esses fatores geram um grande atraso no desenvolvimento dos serviços de saúde, fazendo-se necessárias a efetivação e a participação ativa dos determinantes sociais de saúde no PSF, além da melhor estruturação da política de Estado com base no financiamento, capacitação dos profissionais para realizar as atividades e avaliação com a integração das atividades realizadas em outros níveis de complexidade e gestão (Dowbor \& Westphal, 2013).

Outras fragilidades elencadas foram a falha de cobertura da ESF e a falta de profissionais, como é possível observar nas seguintes falas.

"a maior dificuldade é que só estamos com essa unidade. Quando o 24 h estava funcionando, era melhor, porque ele é no centro e também la atende os ribeirinhos..., o problema é que ele não comporta toda a população de Alter... Acredito que seja o fato da ESF não abranger todas as áreas de alter... tem muita área descoberta”.

"acho que falta mais profissionais”

“... acho que poderia ter mais médicos pra atender a gente” 
Pelo exposto acima, consideram-se necessárias melhorias na construção, reforma e ampliação de ESF, mas, infelizmente, as restrições impostas ao SUS e as políticas sociais pela Emenda Constitucional de 95 ameaçam o desenvolvimento de uma política pública de expansão de insumos (Brasil, 2017).

\section{Conclusão}

Ao término desse estudo, podemos concluir que o primeiro local de procura por assistência à saúde pelos usuários, é a UBS ou Prontos Atendimentos. Ainda em relação aos itinerários praticados pelos usuários, observou-se que a maioria recebe orientações de amigos, familiares e vizinhos para obter a cura por meio de ervas medicinais, espiritualidade, alimentação e até medicações. Foi possível perceber que as potencialidades estão relacionadas a dinâmica de atendimento dos usuários relacionada com a proximidade geográfica da UBS. Logo, sob a perspectiva deste estudo, tem-se que os Itinerários terapêuticos se dão de maneira plural e ao mesmo templo singular, ou seja, próprio de cada indivíduo. Este baseado nas próprias experiências e crenças. Sob a ótica da atenção básica, nota-se que a Unidade Básica de Saúde e a Atenção Primária são referências em atendimento em saúde para as populações estudadas, no entanto a população ainda anseia por melhorias e consegue identificar as potencialidades e fragilidades de suas unidades.

\section{Referências}

Albuquerque, M. D. S. V. D., Lima, L. P., Costa, A. M., \& Melo Filho, D. A. D. (2013). Regulação Assistencial no Recife: possibilidades e limites na promoção do acesso. Saúde e Sociedade, 22, 223-236.

Alves, P. C. B., \& Souza, I. M. (1999). Escolha e avaliação de tratamento para problemas de saúde: considerações sobre o itinerário terapêutico. Experiência de doença e narrativa, 1, 125-38.

Arakawa, A. M., Lopes-Herrera, S. A., Caldana, M. D. L., \& Tomita, N. E. (2012). Percepção dos usuários do SUS: expectativa e satisfação do atendimento na Estratégia de Saúde da Família. Revista CEFAC, 14, 1108-1114.

Ascari, R. A., Ferraz, L., Buss, E., Rennau, L. R., \& Brum, M. L. B. (2014). Estratégia saúde da família: automedicação entre os usuários. Revista Uningá review, 18(2).

Bardin, L.(2011). Análise de conteúdo. São Paulo: Edições 70.

Barros, M. M. A. F., Mendes, M. D. L. C., Frota, L. M. A., \& de Sousa Almeida, J. R. (2018). Acolhimento em unidade de atenção primária à saúde: potencialidades e desafios. SANARE-Revista de Políticas Públicas, 17(2).

Bizinelli, B. M., Neto, P. P., de Albuquerque, G. S. C., Conde, R. E. S., \& Scarin, F. C. (2019). Acesso à Atenção Primária à Saúde em Curitiba: a percepção dos usuários que frequentam uma unidade de pronto atendimento (UPA). APS EM REVISTA, 1(3), 198-205.

Brasil (2017). Política Nacional de Atenção Básica. Portaria n. 2.436, de 21 de setembro de 2017. Aprova a Política Nacional de Atenção Básica, estabelecendo a revisão de diretrizes para a organização da Atenção Básica, no âmbito do Sistema Único de Sa.de (SUS). Diário Oficial da União, Brasília, DF.

Cabral, A. L. L. V., Martinez-Hemáez, A., Andrade, E. I. G., \& Cherchiglia, M. L. (2011). Itinerários terapêuticos: o estado da arte da produção científica no Brasil. Ciência \& Saúde Coletiva, 16, 4433-4442.

Carlomagno, M. C. (2018). Conduzindo pesquisas com questionários online: Uma Introdução as Questões Metodológicas. Estudando cultura e comunicação com mídias sociais, 31 .

Corrêa, A. C. P., Ferreira, F., Cruz, G. S. P., \& Pedrosa, I. C. F. (2011). Acesso a serviços de saúde: olhar de usuários de uma unidade de saúde da família. Revista Gaúcha de Enfermagem, 32, 451-457.

Dowbor, T. P., \& Westphal, M. F. (2013). Determinantes sociais da saúde e o Programa Saúde da Família no município de São Paulo. Revista de Saúde Pública, 47, 781-790.

Fernandes, A. M., Bruchêz, A., d'Ávila, A. A. F., Castilhos, N. C., \& Olea, P. M. (2018). Metodologia de pesquisa de dissertações sobre inovação: Análise bibliométrica. Desafio online, 6(1).

Formiga, B. G. (2016). Programa Saúde da Família, as potencialidades e limitações para o Sistema Único de Saúde: uma revisão integrativa.

Guedes, J. D. S., Santos, R. M. B. D., \& Di Lorenzo, R. A. V. (2011). A implantação do Programa de Saúde da Família (PSF) no Estado de São Paulo (19952002). Saúde e Sociedade, 20, 875-883. 
Research, Society and Development, v. 10, n. 14, e16101421400, 2021

(CC BY 4.0) | ISSN 2525-3409 | DOI: http://dx.doi.org/10.33448/rsd-v10i14.21400

Guerin, GD, Rossoni, E., \& Bueno, D. (2012). Itinerários terapêuticos de usuários de medicamentos de uma unidade de Estratégia de Saúde da Família. Ciência \& Saúde Coletiva , 17 , 3003-3010.

Instituto brasileiro de geografia e estatística[IBGE]. Dados da Cidade de Santarém - Pará. Brasília, DF, 2021.

Kleinman, A. (1980). Pacientes e curandeiros no contexto da cultura . Imprensa da Universidade da Califórnia.

Maliska, I. C. A., \& de Souza Padilha, M. I. C. (2007). AIDS: a experiência da doença e a construção do itinerário terapêutico. Revista Eletrônica de Enfermagem, 9(3).

Marin, M. J. S., Moracvick, M. Y. A. D., \& Marchioli, M. (2014). Acesso aos serviços de saúde: comparação da visão de profissionais e usuários da atenção básica [Health service access: comparing professionals' and users' views of primary care]. Revista Enfermagem UERJ, 22(5), 629-636.

Mendes, E. V. (2010). As redes de atenção à saúde. Ciência \& saúde coletiva, 15, 2297-2305.

Merino, MDFGL e Marcon, SS (2007). Concepções de saúde e itinerário terapêutico adotado por adultos de um município de pequeno porte. Revista Brasileira de Enfermagem, 60, 651-658.

Milanez, TCM, Soratto, J., Ferraz, F., Vitali, MM, Tomasi, CD, Sorato, MT, \& Bittencourt, LTG (2018). Satisfação e insatisfação na Estratégia Saúde da Família: potencialidades a serem exploradas, fragilidades a serem dirimidas. Cadernos Saúde Coletiva, 26, 184-190.

Minayo, M. C. S. (2010). O desafio do conhecimento: metodologia de pesquisa social em saúde. Rio de Janeiro: Editora Hucitec.

Paes, K. D. S. M., Nascimento, J. C., \& Oliveira Negrini, L. D. (2018). O uso da atenção intermediária como porta de entrada preferencial ao sus: a percepção dos usuários classificados como não urgentes na upa 24 horas dr. Valdir de camargo, bragança paulista, sp. Ensaios USF, 2(2), 1-13.

Temer, A., \& Tuzzo, S. A. (2017). A entrevista como método de pesquisa qualitativa: uma Leitura Crítica das memórias dos jornalistas. CIAIQ $2017,3$.

Santos, K. T. D., Saliba, N. A., Moimaz, S. A. S., Arcieri, R. M., \& Carvalho, M. D. L. (2011). Agente comunitário de saúde: perfil adequado a realidade do Programa Saúde da Família?. Ciência \& Saúde Coletiva, 16, 1023-1028.

Santos, D. D. S., Tenório, E. D. A., Brêda, M. Z., \& Mishima, S. M. (2014). Processo saúde/doença e estratégia de saúde da família: o olhar do usuário. Revista Latino-Americana de Enfermagem, 22, 918-925.

Secretaria municipal de saúde de santarém [SEMSA] Informações de Saúde do Município de Santarém.. Santarém, PA, 2020.

Visentin, A., \& Lenardt, MH (2010). O itinerário terapêutico: história oral de idosos com câncer. Acta paulista de enfermagem , 23 , $486-492$. 